\title{
Correction to: Use of Genetic Testing for Primary Immunodeficiency Patients
}

\author{
Jennifer R. Heimall ${ }^{1}$ • David Hagin ${ }^{2}$ • Joud Hajjar ${ }^{3}$ - Sarah E. Henrickson ${ }^{4,5}$ • Hillary S. Hernandez-Trujillo ${ }^{6,7}$. \\ Yuval Itan ${ }^{8,9} \cdot$ Lisa Kobrynski $^{10}$. Kenneth Paris ${ }^{11}$ - Troy R. Torgerson ${ }^{12} \cdot$ James W. Verbsky $^{13}$.

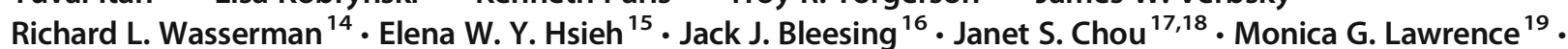 \\ Rebecca A. Marsh ${ }^{20}$ - Sergio D. Rosenzweig ${ }^{21}$ • Jordan S. Orange $22,23 \cdot$ Roshini S. Abraham $^{24}$
}

Published online: 21 May 2018

(C) Springer Science+Business Media, LLC, part of Springer Nature 2018

\section{Correction to: J Clin Immunol. 2018 Apr 19} https://doi.org/10.1007/s10875-018-0489-8

The original version of this article unfortunately contained mistakes in some of the author names and affiliations. The correct list of author names and affiliations is below, with the corrections in bold:

Jennifer R Heimall, MD: Division of Allergy and Immunology, Children's Hospital of Philadelphia; University of Pennsylvania.

David Hagin, MD, PhD: Allergy and Immunology Division, Tel-Aviv Sourasky Medical Center.

Joud Hajjar, MD, MS: Department of Pediatrics, Section of Immunology, Allergy and Rheumatology, Baylor College of Medicine.

Sarah E Henrickson, MD, PhD: 1. University of Pennsylvania, Division of Allergy and Immunology, Children's Hospital of Philadelphia, Philadelphia, PA 2. University of Pennsylvania, Institute for Immunology, Philadelphia, PA.

Hillary S Hernandez- Trujillo, MD MSCE: Division of Infectious Disease \& Immunology, Connecticut Children's Medical Center, Hartford, CT and CT Asthma and Allergy Center, West Hartford, CT.

Yuval Itan, PhD: 1. The Charles Bronfman Institute for Personalized Medicine, Icahn School of Medicine at Mount

The online version of the original article can be found at https://oi.org/ 10.1007/s10875-018-0489-8

Jennifer R. Heimall

heimallj@email.chop.edu

Extended author information available on the last page of the article
Sinai, New York, NY, USA 2. Department of Genetics and Genomic Sciences, Icahn School of Medicine at Mount Sinai, New York, NY, USA.

Lisa Kobrynski, MD, MPH: Associate Professor of Pediatrics, Marcus Professor of Immunology, Section, Allergy/Immunology, Emory University.

Kenneth Paris, MD, MPH: Division of AllergyImmunology, LSU Health Sciences Center, Children's Hospital.

Troy R Torgerson, MD, PhD: Department of Pediatrics, University of Washington.

James W Verbsky, MD, PhD: Associate Professor of Pediatrics and Microbiology and Molecular Genetics Section of Pediatric Rheumatology, Medical College of Wisconsin.

Richard L Wasserman, MD, PhD: Department of Pediatrics, Medical City Children's Hospital.

Elena WY Hsieh, MD: Department of Immunology and Microbiology, Department of Pediatrics, Division of Allergy and Immunology, University of Colorado, School of Medicine.

Jack J Bleesing, MD, PhD: Professor, Division of Bone Marrow Transplantation and Immune Deficiency, Cancer and Blood Diseases Institute, Cincinnati Children's Hospital Medical Center.

Janet S Chou, MD: Division of Immunology, Boston Children's Hospital; Department of Pediatrics, Harvard Medical School, Boston.

Monica G Lawrence, MD: Division of Asthma, Allergy and Clinical Immunology, University of Virginia Health System.

Rebecca A Marsh, MD: Bone Marrow Transplantation and Immune Deficiency, Cancer and Blood Diseases Institute, Cincinnati Children's Hospital Medical Center, Cincinnati, $\mathrm{OH}$.

Sergio D Rosenzweig, MD, PhD: NIH Clinical Center, NIH. 
Jordan S Orange, MD, PhD: Chief, Immunology, Allergy and Rheumatology, Director, Center for Human Immunobiology, Texas Children's Hospital; Professor of Pediatrics, Pathology and Immunology, Vice Chair for Research, Department of Pediatrics, Baylor College of Medicine.
Roshini S. Abraham, PhD: Department of Laboratory Medicine and Pathology, Mayo Clinic, Rochester, MN.

Thank you

Jennifer Heimall, MD

\section{Affiliations}

Jennifer R. Heimall ${ }^{1}$ • David Hagin ${ }^{2}$ • Joud Hajjar ${ }^{3}$ • Sarah E. Henrickson ${ }^{4,5} \cdot$ Hillary S. Hernandez-Trujillo ${ }^{6,7}$. Yuval Itan ${ }^{8,9} \cdot$ Lisa Kobrynski ${ }^{10} \cdot$ Kenneth Paris ${ }^{11} \cdot$ Troy R. Torgerson $^{12} \cdot$ James W. Verbsky ${ }^{13} \cdot$ Richard L. Wasserman $^{14}$. Elena W. Y. Hsieh ${ }^{15}$ • Jack J. Bleesing ${ }^{16}$ • Janet S. Chou ${ }^{17,18}$ • Monica G. Lawrence ${ }^{19} \cdot$ Rebecca A. Marsh $^{20}$ • Sergio D. Rosenzweig ${ }^{21} \cdot$ Jordan S. Orange ${ }^{22,23} \cdot$ Roshini S. Abraham ${ }^{24}$

1 Division of Allergy and Immunology, Children's Hospital of Philadelphia, University of Pennsylvania, Wood Building 3rd Floor, 3401 Civic Center Blvd, Philadelphia, PA 19104, USA

2 Allergy and Immunology Division, Tel-Aviv Sourasky Medical Center, Tel-Aviv, Israel

3 Department of Pediatrics, Section of Immunology, Allergy and Rheumatology, Baylor College of Medicine, Houston, TX, USA

4 Division of Allergy and Immunology, Children's Hospital of Philadelphia, University of Pennsylvania, Philadelphia, PA, USA

5 Institute for Immunology, University of Pennsylvania, Philadelphia, PA, USA

6 Division of Infectious Disease \& Immunology, Connecticut Children's Medical Center, Hartford, CT, USA

7 CT Asthma and Allergy Center, Hartford, West, CT, USA

8 Icahn School of Medicine at Mount Sinai, The Charles Bronfman Institute for Personalized Medicine, New York, NY, USA

9 Department of Genetics and Genomic Sciences, Icahn School of Medicine at Mount Sinai, New York, NY, USA

10 Allergy/Immunology, Emory University, Atlanta, GA, USA

11 Division of Allergy-Immunology, LSU Health Sciences Center, Children's Hospital, New Orleans, LA, USA

12 Department of Pediatrics, University of Washington, Seattle, WA, USA

13 Pediatrics and Microbiology and Molecular Genetics Section of Pediatric Rheumatology, Medical College of Wisconsin, Milwaukee, WI, USA
14 Department of Pediatrics, Medical City Children's Hospital, Dallas, TX, USA

15 Department of Immunology and Microbiology, Department of Pediatrics, Division of Allergy and Immunology, University of Colorado, School of Medicine, Aurora, CO, USA

16 Division of Bone Marrow Transplantation and Immune Deficiency, Cancer and Blood Diseases Institute, Cincinnati Children's Hospital Medical Center, Cincinnati, OH, USA

17 Division of Immunology, Boston Children's Hospital, Boston, MA, USA

18 Department of Pediatrics, Harvard Medical School, Boston, MA, USA

19 Division of Asthma, Allergy and Clinical Immunology, University of Virginia Health System, Charlottesville, VA, USA

20 Bone Marrow Transplantation and Immune Deficiency, Cancer and Blood Diseases Institute, Cincinnati Children's Hospital Medical Center, Cincinnati, $\mathrm{OH}$, USA

21 NIH Clinical Center, Bethesda, MD, USA

22 Immunology, Allergy and Rheumatology, Director, Center for Human Immunobiology, Texas Children's Hospital, Houston, TX, USA

23 Department of Pediatrics, Baylor College of Medicine, Houston, TX, USA

24 Department of Laboratory Medicine and Pathology, Mayo Clinic, Rochester, MN, USA 